\title{
Le corpus de la sculpture de Cluny
}

N. STRATFORD (dir.), Corpus de la sculpture de Cluny. Les parties orientales de la Grande Église Cluny III, 2 vol., Paris, éd. Picard, 2011, 824 p., 1436 ill. (photographies, plans et dessins).

\section{Neil Stratford}

\section{(2) OpenEdition} Journals

\section{Édition électronique}

URL : https://journals.openedition.org/cem/12077

DOI : $10.4000 /$ cem. 12077

ISSN : 1954-3093

Éditeur

Centre d'études médiévales Saint-Germain d'Auxerre

Édition imprimée

Pagination : 463-465

ISSN : 1623-5770

\section{Référence électronique}

Neil Stratford, "Le corpus de la sculpture de Cluny », Bulletin du centre d'études médiévales d'Auxerre I BUCEMA [En ligne], 15 | 2011, mis en ligne le 25 août 2011, consulté le 22 septembre 2022. URL http://journals.openedition.org/cem/12077 ; DOI : https://doi.org/10.4000/cem.12077

Ce document a été généré automatiquement le 22 septembre 2022.

\section{(c) (i) $\$$ (O)}

Creative Commons - Attribution - Pas d'Utilisation Commerciale - Partage dans les Mêmes Conditions 4.0 International - CC BY-NC-SA 4.0

https://creativecommons.org/licenses/by-nc-sa/4.0/ 


\section{Le corpus de la sculpture de Cluny}

N. STRATFORD (dir.), Corpus de la sculpture de Cluny. Les parties orientales de la Grande Église Cluny III, 2 vol., Paris, éd. Picard, 2011, 824 p., 1436 ill. (photographies, plans et dessins).

\section{Neil Stratford}

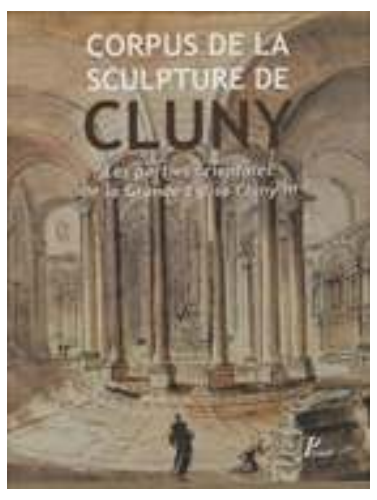

1 Il n'est pas nécessaire de rappeler ici les circonstances qui ont voué Cluny III à la pioche des démolisseurs entre 1792 et 1820 . Depuis 1988, les recherches ont été menées à Cluny par l'équipe de chercheurs afin de publier, non seulement, les vestiges encore conservés in situ dans les parties subsistantes de l'édifice, mais aussi les fragments sauvegardés lors de la destruction et conservés au musée Ochier, ainsi que ceux qui ont été découverts en fouille par l'américain Kenneth John Conant entre 1928 et 1950. C'est K. J. Conant lui-même qui m'a demandé, peu avant sa mort, de publier ses découvertes en matière de sculpture. Le travail s'est développé à un rythme inégal. Les difficultés liées aux conditions de stockage du matériel étudié et aux sujétions financières ont contribué à la longue gestation d'un ouvrage, que nous avons conçu comme un outil de travail.

2 Le catalogue de l'important ensemble de fragments (plusieurs milliers) trouvés par $\mathrm{K}$. J. Conant a été organisé suivant l'ordre des sondages de K. J. Conant, qu'il désignait sous le nom de «Pits». Il va sans dire que les contraintes avec lesquelles K. J. Conant dut composer, comme la présence de bâtiments construits sur l'emplacement de l'église, notamment ceux du haras national, installé à Cluny depuis 1811, nous laissent 
sur notre faim pour une grande partie du site. En revanche, une évidence a été confirmée depuis le début de nos recherches: pendant les démolitions qui se poursuivirent pendant trente ans après la Révolution, les petits fragments, qui ne présentaient pas d'intérêt pour les démolisseurs, restèrent plus ou moins sur place, non loin de leur cadre d'origine. Il en existe de multiples preuves, comme les fragments provenant d'un seul et même chapiteau, souvent découverts les uns à côté des autres. K. J. Conant a soigneusement identifié les fragments en les marquant d'un numéro et de la date de la trouvaille, qui renvoient aux cahiers des fouilles conservés au musée Ochier, accompagnés d'une riche documentation iconographique (photographies et dessins). Les vestiges sculptés sont souvent fragmentaires, beaucoup sont de simples moulures - les éléments figurés sont en effet assez rares. Aussi, le catalogue repose-t-il en large partie sur les minutieux dessins de David Walsh, les pièces les plus parlantes étant toutefois reproduites aussi en photographies. Vingt-six Pits sont ainsi répertoriés, ce qui correspond au total à environ 2350 fragments, couvrant toutes les périodes depuis la fondation de la grande église en 1088 jusqu'au XVIII ${ }^{e}$ siècle. L'époque romane est sans doute quantitativement la plus importante, tant par la sculpture monumentale que par le mobilier liturgique. Les périodes gothiques et modernes sont, elles aussi, richement représentées.

3 La première partie est une introduction au catalogue. David Walsh analyse l'approche archéologique de K.J. Conant replacée dans son temps. Nous présentons ensuite l'abondante documentation médiévale, moderne et post-révolutionnaire, intéressant Cluny III, sa construction, les interventions des époques gothique et néoclassique, la longue période de démolition et, enfin, les changements et les restaurations des $\mathrm{XIX}^{\mathrm{e}}$ et $\mathrm{xx}^{\mathrm{e}}$ siècles. La complexité d'un tel ouvrage et sa longueur nous ont conduits à omettre toute référence aux derniers travaux de restauration de Cluny, ainsi qu'aux récentes fouilles, liées aux célébrations en $2010 \mathrm{du} \mathrm{XI}{ }^{\mathrm{e}}$ centenaire de la fondation de Cluny. Les volumes à venir feront le point sur ces nouveaux développements documentaires et bibliographiques.

4 Toujours en introduction, nous présentons le catalogue raisonné de l'iconographie de la grande église, avant et après sa démolition. Il s'agit d'un répertoire le plus complet possible, comprenant certaines images nouvellement découvertes, même si les fonds des collections, surtout les collections privées, n'ont pas encore tout révélé. Vient ensuite le catalogue des tombeaux de Cluny que nous avons établi d'après les descriptions - surtout des $\mathrm{XVII}^{\mathrm{e}}, \mathrm{XVIII}^{\mathrm{e}}$ et $\mathrm{XIX}^{\mathrm{e}}$ siècles. Cluny était une importante nécropole, la grande église et le monastère abritaient de très nombreux tombeaux des abbés et des grands dignitaires de Cluny, mais aussi d'un pape, de cardinaux, d'archevêques, d'évêques et de grands laïques - la perte presque totale de ces monuments funéraires n'est hélas pas compensée par des croquis. Cluny ne figurait pas, semble-t-il, dans la collection de Gaignières, et celle de l'érudit dijonnais Pierre Palliot fut détruite par un incendie au $\mathrm{XVIII}^{\mathrm{e}}$ siècle. La partie introductive du livre se termine avec un très important chapitre d'Annie Blanc sur les matériaux de construction employés à Cluny, avec, lorsque cela est possible, une cartographie des carrières. Une recherche de cette nature, menée par un géologue sur un monument grand et complexe, était pionnière lorsqu'elle a commencé ses analyses et ses prospections de terrain, dans les années 1980.

5 Le catalogue des fragments, qui représente le cœur du livre, est suivi par des sections indispensables pour la compréhension du décor de Cluny III. Ce qui subsiste des parties 
orientales de l'église est étudié en premier lieu, qu'il s'agisse de l'abbatiale romane tous les chapiteaux in situ sont décrits et illustrés - ou des chapelles gothiques, dont les plus importantes sont la chapelle Saint-Martial, chef-d'œuvre de l'architecture rayonnante parisienne, construite pour l'abbé Pierre II de Chastellux (1322-1342, $\dagger 1344$ ), et la chapelle de Jean de Bourbon (abbé de Cluny, 1456/1457-1480, †1485). Celle-ci, avec ses magnifiques sculptures attribuées à Antoine Le Moiturier, est étudiée en détail par Brigitte Maurice-Chabard.

6 Un chapitre entier est dédié à la grande abside de Cluny III. Les colonnes antiques en marbre, qui avaient été remployées dans l'hémicycle, sont analysées par Annie Blanc et le laboratoire du British Museum. Nous avons consacré une section aux huit grands chapiteaux du rond-point, en abordant le problème de l'identité de leurs figures (dans certains cas) et de leurs inscriptions, ainsi que celui de leur style et de leur influence, en présentant un dossier sur plusieurs monuments dans lesquels l'équipe de sculpteurs du rond-point a travaillé - non seulement en Bourgogne (à Vézelay, par exemple), mais plus au sud (Avenas, Romans, Saint-Paul-de-Varax, Savigny). Les descriptions connues de la grande peinture, qui décorait le cul-de-four de l'abside, sont également transcrites.

7 Suit un chapitre sur le mobilier liturgique, d'après les documents, avec, surtout, une étude de la clôture romane du chœur des moines, dont environ cent fragments ont été identifiés. Cet ensemble existait déjà vers 1120-1121, d'après un passage de la Vie de saint Hugues, écrite par le moine Gilon pour l'abbé Pons de Melgueil, le grand successeur de saint Hugues (1109-1122, † 1126). Selon l'étude de David Walsh et ses reconstitutions graphiques, cette clôture formait une haute barrière. Cette découverte bouleverse la présomption, si souvent énoncée par les spécialistes de la liturgie, que les hautes clôtures n'apparurent que plus tard, en préambule aux jubés gothiques. La haute clôture de Cluny est la plus ancienne connue jusqu'à présent : cette nouvelle tendance était-elle une réponse liturgique aux besoins de la "réforme Grégorienne »?

8 Quelques tombeaux de Cluny subsistent en partie. Celui du fondateur de la Grande Église, saint Hugues, nous a légué quelques très beaux fragments, qui aident à une restitution partielle par Jean-Denis Salvèque et David Walsh, de cet insigne monument, érigé derrière l'autel matutinal, sans doute au moment de la « canonisation » du grand abbé vers 1120. Vincent Droguet présente l'histoire et les sculptures du mausolée que le cardinal de Bouillon projetait d'installer pour sa famille dans le bras nord du petit transept, à l'extrême fin du xVII siècle. Ces sculptures, exécutées à Rome par Pierre II Legros, comptent parmi les plus grands chefs-d'œuvre de la sculpture baroque française ; elles sont miraculeusement conservées à l'hôpital de Cluny.

9 Les parties du livre qui concernent la technique des peintures murales et la polychromie des sculptures (Juliette Rollier-Hanselmann), les dallages et mosaïques de pavements romans (Brigitte Maurice-Chabard), les marbres décoratifs (Annie Blanc) et les carreaux vernissés des pavements médiévaux (Matthieu Pinette) ne sont pas les moins importantes. 
INDEX

Index géographique : France/Cluny

Mots-clés : corpus, sculpture 